\title{
ABI SOLiD Sequencing
}

National Cancer Institute

\section{Source}

National Cancer Institute. ABI SOLiD Sequencing. NCI Thesaurus. Code C146811.

A proprietary next-generation DNA sequencing system from Applied Biosystems that utilizes DNA ligase. DNA fragments attached to beads are clonally amplified and ligated to fluorescently labeled "interrog ation probes". Ligation frees the fluor, which is detected and identifies the specific probe. The marker of the attached probe is then removed and a 5'-phosphate group is regenerated. Multiple cycles of ligation, detection, and cleavage are performed. 